\title{
Searches for possible T-odd and P-odd short range interactions using polarized nuclei
}

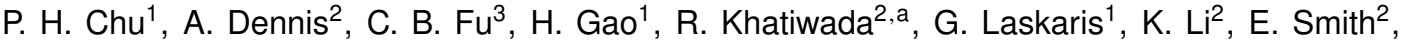 \\ W. M. Snow ${ }^{2}$, H. Yan ${ }^{2}$, and W. Zheng ${ }^{1}$ \\ ${ }^{1}$ Triangle Universities Nuclear Laboratory and Department of Physics, Duke University, Durham, North Car- \\ olina 27708, USA \\ ${ }^{2}$ Department of Physics, Indiana University, Bloomington, Indiana 47408, USA \\ ${ }^{3}$ Department of Physics, Shanghai Jiaotong University, Shanghai, 200240, China.
}

\begin{abstract}
Various theories predict the possible existence of T-odd and P-odd shortrange forces between spin $\frac{1}{2}$ fermions, proportional to $\mathbf{S} \cdot \mathbf{r}$ where $\mathbf{S}$ is the fermion spin and $\mathbf{r}$ is the separation between particles. We use ensembles of polarized nuclei and an un-polarized mass to search for such a force over sub-mm ranges. We established an improved upper bound on the product $g_{s} g_{p}^{n}$ of the scalar coupling to particles in the un-polarized mass and the pseudo-scalar coupling of polarized neutrons for force ranges from $10^{-4}$ to $10^{-2} \mathrm{~m}$, corresponding to a mass range of $2 \cdot 10^{-3}$ to $2 \cdot 10^{-5} \mathrm{eV}$ for the exchange boson [1].
\end{abstract}

Possible short-range $(\mathrm{mm}-\mu \mathrm{m})$ forces between polarized spin $\frac{1}{2}$ particles and un-polarized masses may exist beyond the Standard Model [2]. In particular a so-called monopole-dipole interaction would couple to fermions through scalar and pseudoscalar vertices by the exchange of spin-0 bosons [3]. This force has a Yukawa-type of interaction potential from one boson exchange

$$
V(r)=\frac{g_{s} g_{p}^{n} \hbar^{2}}{8 \pi m_{p}}(\hat{\sigma} \cdot \hat{r})\left(\frac{1}{r \lambda}+\frac{1}{r^{2}}\right) \exp ^{\frac{-r}{\lambda}}
$$

where $\hat{r}$ is the unit vector from the polarized particle to the unpolarized particle, $\hat{\sigma}$ is the spin of the polarized particle, $m_{p}$ is the mass of the polarized particle, $g_{s} g_{p}^{n}$ denotes the product of the couplings of the scalar vertex in the unpolarized mass and pseudoscalar vertex in the polarized mass and $\lambda$ is the force range. Such a P-odd and T-odd interaction proportional to $\vec{\sigma} \cdot \vec{r}$ can cause a shift in the precession frequency of the polarized particle in the presence of an unpolarized mass $[4,5]$. This experiment presents improved laboratory limits on $g_{s} g_{p}^{n}$ in comparison to a previous work [6] for force ranges from $10^{-4}$ to $10^{-2} \mathrm{~m}$. These results can be interpreted in terms of the pseudoscalar coupling $g_{p}^{n}$, since the spin of the polarized ${ }^{3} \mathrm{He}$ used in this work is dominated by the spin of its constituent neutron [7]. The pseudoscalar coupling constant from the polarized mass is spin dependent whereas the scalar coupling constant from the unpolarized mass is spin independent but fermion density dependent. This work also represents to our knowledge the most sensitive measurement done with ${ }^{3} \mathrm{He}$ at low energies for $\mathrm{T}$ and $\mathrm{P}$ odd interactions.

\footnotetext{
a e-mail: rakhatiw@indiana.edu
} 
Spin Exchange Optical Pumping (SEOP) system was used to polarize ${ }^{3} \mathrm{He}$. A 7 amg high-pressure ${ }^{3} \mathrm{He}$ glass cell with $250 \mu \mathrm{m}$ thick hemispherical glass windows at both ends based on the design of Ref. [6] was used. Two identical NMR pickup coils A and B were used to detect the possible precession frequency shift of the polarized ${ }^{3} \mathrm{He}$ nuclei due to the presence of the unpolarized mass and to simultaneously monitor the magnetic holding field. To account for any holding field drift, measured frequency in coil B was subtracted from A. The Helmholtz coil current produced a $23.8 \mathrm{kHz}$ Larmor frequency for ${ }^{3} \mathrm{He}$. A $24-\mathrm{kHz}$ RF pulse was applied to tip the spin of the ${ }^{3} \mathrm{He}$. The induced emf in the pickup coil A was recorded as time signal $s(t)$ which was then Fourier transformed into frequency domain, and the real $R(f)$ and imaginary parts $I(f)$ and were numerically calculated using Richardson extrapolation [8]. The resultant frequency domain signal amplitude was $S(f)=\sqrt{R(f)^{2}+I(f)^{2}}$. The precession frequency was determined by locating the maximum of $S(f)$ by varying the reference frequency with a $10^{-6} \mathrm{~Hz}$ step [9].

The experimental technique used two positions of the polarized ${ }^{3} \mathrm{He}$ and the unpolarized mass ensemble. The "mass out" state was defined as the absence of unpolarized mass near the polarized ${ }^{3} \mathrm{He}$ glass cell and the "mass in" state has the mass right next to the cell. The two states were separated by 60 seconds. Two non-magnetic, electrically insulating materials with low magnetic susceptibility were used as the unpolarized test masses, Macor ceramic from Ref. [6] and a 1.02\% Manganese Chloride $\left(\mathrm{MnCl}_{2}\right)$ salt solution in pure water. To avoid systematic errors from the linear and quadratic time dependent drifts in the external magnetic field, we apply an analysis algorithm presented in Ref. [10]. If the frequency with linear and quadratic time dependent drifts is $f(t) \propto a t+b t^{2} \pm c$, where $a, b$ are arbitrary constants, $\pm c$ are frequency shift of mass in/out states, we apply the algorithm,

$$
\Delta f=\frac{1}{4}\left[f_{\text {in }, 1}-3 f_{\text {out }, 1}+3 f_{\text {in }, 2}-f_{\text {out }, 2}\right]
$$

Where $f_{\text {in } / \text { out }, 1 / 2}$ is the difference frequency measured in the pickup coil A-pickup coil B. In/Out denote mass positions \& 1,2 denote the number of cycles. A cycle defines one set of In and Out motions such that,

$$
=\frac{1}{4}\left[\left(a \delta t+b \delta t^{2}+c\right)-3\left(a(2 \delta t)+b(2 \delta t)^{2}-c\right)+3\left(a(3 \delta t)+b(3 \delta t)^{2}+c\right)-\left(a(4 \delta t)+b(4 \delta t)^{2}-c\right)\right]=2 c
$$

$\delta t$ denotes the time step. Four different configurations of holding field direction $(+\mathrm{B} /-\mathrm{B})$ and ${ }^{3} \mathrm{He}$ polarization direction $(+\mathrm{P} /-\mathrm{P})$ give different $\Delta f$ 's in states $+\mathrm{B}+\mathrm{P},+\mathrm{B}-\mathrm{P},-\mathrm{B}+\mathrm{P}$ and $-\mathrm{B}-\mathrm{P}$. Each of these configurations should yield the same magnitude of the frequency shift due to a monopole-dipole interaction. We analyzed two of these configurations $+\mathrm{B}-\mathrm{P}$ and $-\mathrm{B}+\mathrm{P}$ as the other two configurations possessed low $T_{2}$ relaxation time due to residual field gradients. For these configurations,

$$
\begin{aligned}
& f_{+, \pm}=f_{B} \pm \Delta f_{P}+\Delta f_{S D S R F} \\
& f_{-, \mp}=f_{B} \pm \Delta f_{P}-\Delta f_{S D S R F} \\
& \Delta f_{S D S R F}=\frac{1}{2}\left(\Delta f_{+-}-\Delta f_{-+}\right)
\end{aligned}
$$

Where the first and second subscripts in the first two equations' left sides denote the direction of B and $\mathrm{P}$ respectively. $f_{B}$ is the magnetic field dependent precession, which includes contribution from the holding field as well as the possible contribution from the magnetic susceptibility of the mass. $\Delta f_{P}$ is the precession due to the polarization and $\Delta f_{S D S R F}$ is the precession due to the monopole-dipole interaction. $\Delta f_{+-}$and $\Delta f_{-+}$are the frequency differences between the mass in and mass out states and the frequency measured in the pickup coil A minus the pickup coil B of each configuration. The 
Table 1. Average frequency difference between mass-in and mass-out states for the corresponding configurations of $\mathrm{B}$ and $\mathrm{P}$ after subtracting the frequency measured by coil B from coil A.

\begin{tabular}{llll}
\hline sample & $\Delta f_{B, P:+,-}\left(10^{-5} \mathrm{~Hz}\right)$ & $\Delta f_{B, P:-,+}\left(10^{-5} \mathrm{~Hz}\right)$ & $\Delta f_{S D S R F}\left(10^{-5} \mathrm{~Hz}\right)$ \\
\hline Macor Ceramic & $0.6 \pm 1.3$ & $-4.6 \pm 3.1$ & $2.6 \pm 1.7$ \\
$\mathrm{MnCl}_{2}$ Solution & $-3.3 \pm 0.8$ & $-1.7 \pm 5.2$ & $-0.8 \pm 2.6$ \\
\hline
\end{tabular}

uncertainty in the measured frequency shift is given by, $\sigma=\frac{1}{2} \sqrt{\sigma_{+-}^{2}+\sigma_{-+}^{2}}$. For every configuration of each mass, 1000 cycles of data were taken. The data of both the $\mathrm{MnCl}_{2}$ solution and the Macor are shown in Table 1 and the plots of the frequency difference for both are in Fig 1 (left), which show that the average frequency difference for both of these samples are consistent with zero. We use this result to constrain the force range and the coupling strength as shown in Fig 1 (right). The precession frequency shift due to the monpole-dipole interaction for each polarized ${ }^{3} \mathrm{He}$ nucleus in the target chamber was calculated by numerically integrating Eq. (1) over the unpolarized mass as,

$$
\Delta f\left(\vec{z}, \lambda, g_{s} g_{p}^{n}\right)=\frac{2 N}{2 \pi \hbar} \int_{v o l} V(\vec{r}-\vec{z}) d r^{3},
$$

where $N$ is the particle number density of the unpolarized mass, $\vec{z}$ the distance between the surface of the mass and the polarized ${ }^{3} \mathrm{He}$ and vol denotes the volume of the unpolarized mass. For the pickup coil, the precession signal detected is,

$$
s(t) \propto \int\left(\vec{B}_{\text {coil }} \cdot \frac{\partial \vec{M}}{\partial t}\right) d r^{3}
$$

where $\vec{B}_{\text {coil }}$ is the field profile of the pickup coil calculated from [11] and $\vec{M}$ is the magnetization vector of ${ }^{3} \mathrm{He}$. In terms of the precession frequency shift $\Delta f$ and the Larmor frequency $f_{0}$ of ${ }^{3} \mathrm{He}$, the signal can be written as, $s(t)=C\left(\int-B_{x} \cdot\left(f_{0}+\Delta f\right) \sin \left(2 \pi\left(f_{0}+\Delta f\right) t\right)+B_{y} \cdot\left(f_{0}+\Delta f\right) \cos \left(2 \pi\left(f_{0}+\Delta f\right) t\right) d z\right)$, where $f_{0}=\frac{\gamma B_{0}}{2 \pi}$ is the Larmor frequency, $\frac{\gamma}{2 \pi}=-3.24 \mathrm{~Hz} / \mathrm{mG}$ is the gyromagnetic ratio of ${ }^{3} \mathrm{He}, d$ is the ${ }^{3} \mathrm{He}$ glass cell thickness and $C$ is a constant. The signal above can be Fourier transformed into frequency domain power signal, $P\left(f^{\prime}\right)=C\left(\left(\int\left(f_{0}+\Delta f\right) B_{x} \delta\left(f_{0}+\Delta f-f^{\prime}\right) d z\right)^{2}+\left(\int\left(f_{0}+\Delta f\right) B_{y} \delta\left(f_{0}+\Delta f-f^{\prime}\right) d z\right)^{2}\right)$. From this we get the average frequency in the pickup coil, $\bar{f}^{\prime}=\left(\int f^{\prime} P\left(f^{\prime}\right) d f^{\prime}\right) /\left(\int P\left(f^{\prime}\right) d f^{\prime}\right)$ and the desired frequency shift can be obtained from the Larmor frequency $f_{0}$ and the average frequency $\bar{f}^{\prime}$

$$
\Delta \bar{f}\left(\lambda, g_{s} g_{p}^{n}\right)=\bar{f}^{\prime}-f_{0} .
$$

Our frequency differences in mass in and out states are consistent with zero within 1.5 standard deviations as plotted in Fig 1 (left) for both Macor and $\mathrm{MnCl}_{2}$ solution. Our constraint on the coupling constant product $g_{s} g_{p}^{n}$ and the force range is plotted in Fig 1 (right). Our upper bound is a factor of 10-30 improvement compared to previous work corresponding to the mass range of $2 \cdot 10^{-3}$ to 2 . $10^{-5} \mathrm{eV}$ for the pseudoscalar boson and the force range of $10^{-4}$ to $10^{-2} \mathrm{~m}$. More recent results from an Indiana University/Northup Grumman/University of Wisconsin collaboration which used a similar technique of analyzing NMR frequency shifts in a ${ }^{129} \mathrm{Xe}$ and ${ }^{131} \mathrm{Xe}$ co-magnetometer in the presence of zirconia to look for an $\vec{S} \cdot \vec{r}$ interaction over similar range [12] is also included in Fig 1 (right). By comparing the frequency shifts in these two species of Xe, one can distinguish between the frequency change due to a monopole-dipole interaction and the background magnetic field changes. Another recent constraint from Ref [13] which looked for $\vec{S} \cdot \vec{r}$ interaction over similar range is also plotted in Fig 1 (right). 

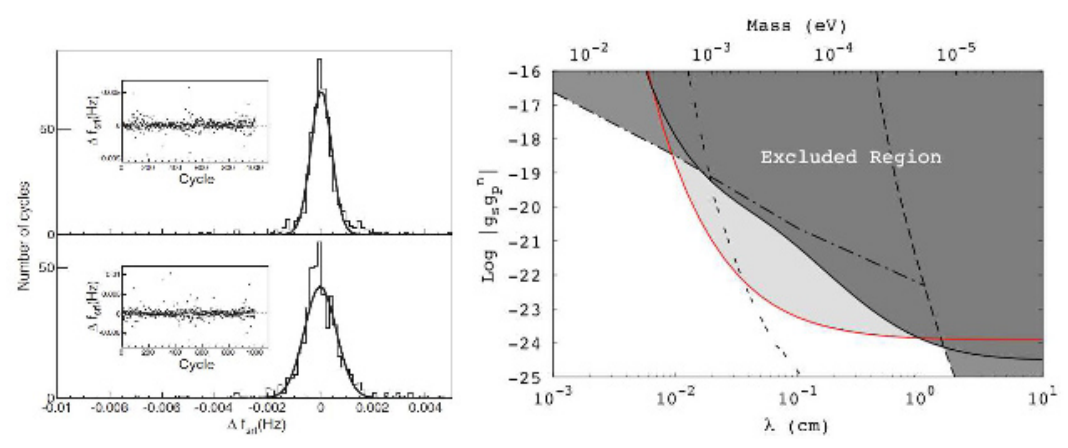

Figure 1. (Left) Plots of frequency differences for Macor ceramic (top) and $\mathrm{MnCl}_{2}$ solution (bottom) for 1000 cycles. The average $\Delta f_{S D S R F}$ is consistent with zero for both. (Right) Constraints on the coupling constant product $g_{s} g_{p}^{n}$ as a function of the force range $\lambda$ and corresponding mass of the exchange boson. The dashed curve is from [4], dash-dotted from [14], solid black from [1], solid red (below) from [12] and double dash from [13].

We plan to concentrate in future work on the shorter range $\left(<10^{-3} \mathrm{~m}\right)$ region with future improvements in the sensitivity of this experiment. We plan to use a thinner glass window for the polarized ${ }^{3} \mathrm{He}$ cell, a more uniform holding field, improved magnetic shielding, and higher density test masses. Special thanks to M. Souza, T. Averett, Y. Zhang, S. Jawalkar, T. Gentile, M. Yu. Khlopov and P. Fayet for help with the appratus and useful insight regarding the experiment. This work was supported by Duke University, the U.S. Department of Energy under Contract DE-FG02-03ER41231, and the U.S. National Science Foundation through Grant No. PHY-1068712. The work of K. Li, R. Khatiwada, E. Smith, W. M. Snow, and H. Yan was supported in part by the Indiana University Center for Spacetime Symmetries and A. Dennis by the IU STARS program.

\section{References}

[1] P. H. Chu, A. Dennis, C. B. Fu, H. Gao, R. Khatiwada, G. Laskaris, K. Li, E. Smith, W. M. Snow, H. Yan, and W. Zheng, Phys. Rev. D 87, 011105(R) (2013).

[2] J. Leitner and S. Okubo, Phys. Rev. B 136, B1542 (1964).

[3] J. E. Moody and F. Wilczek, Phys. Rev. D 30, 130 (1984).

[4] A. N. Youdin et al., Phys. Rev. Lett. 77, 2170 (1996).

[5] W. -T. Ni, S. -S. Pan, H. -C. Yeh, L. -S. Hou, and J. Wan, Phys. Rev. Lett. 82, 2439 (1999).

[6] W. Zheng, et al., Phys. Rev. D 85, 031505 (2012).

[7] J. L. Friar, et al., Phys. Rev. C 42, 2310 (1990).

[8] L. F. Richardson, Phil. Trans. R. Soc. A 210, 307 (1911); L. F. Richardson and J. A. Gaunt, Phil. Trans. R. Soc. A 226, 299 (1927).

[9] H. Yan et al. Accepted in Communications in Computational Physics (CiCP).

[10] H. E. Swanson and S. Schlamminger, Meas. Sci. Technol. 21, 115104 (2010).

[11] E. K. Insko, M. A. Elliott, J. C. Schotland, and J. S. Leigh, J. Magn. Reson. 131, 111 (1998).

[12] M. Bulatowicz, R. Griffith, M. Larsen, J. Mirijanian, C. B. Fu, E. Smith, W. M. Snow, H. Yan, and T. G. Walker, Phys. Rev. Lett. 111, 102001 (2013).

[13] K. Tullney et al., Phys. Rev. Lett. 111, 100801 (2013).

[14] A. Petukhov, G. Pignol, D. Jullien, and K. Andersen, Phys. Rev. Lett. 105, 3 (2010). 